\title{
Acoustic forcing on swirling flow: experiments and simulation
}

\author{
W. Hubschmid $\cdot$ A. Denisov $\cdot$ F. Biagioli
}

Received: 20 February 2014/Revised: 28 July 2014/Accepted: 30 July 2014/Published online: 20 August 2014

(C) Springer-Verlag Berlin Heidelberg 2014

\begin{abstract}
We investigated the effect of sound irradiated from loudspeakers on the flow of preheated air in the combustion chamber of a swirl burner. The temporally periodic pattern of the flow generated by the sound was detected by fast particle image velocimetry (PIV), with a repetition rate that was adapted to the observation of 12 phase angles of the irradiated monochromatic sound. The strong observed movement of the air is related to the movement by the sound itself, as determined by the pressure measurements with microphones. The PIV measurements reveal also a nonlinear interaction between the irradiated sound and the precession of the vortex core. The accuracy of the sound measurements was tested by determining in quiescent air the acoustic velocity by microphones and as well by PIV; good agreement was obtained thereby. Numerical calculations, using large eddy simulation and accounting for the sound forcing by variation in the mass flow at the inlet of the computational domain, approximately reproduce some of the experimental results.
\end{abstract}

\section{Introduction}

The flow in swirl-stabilized combustion downstream the burner exhibits strong periodic variations at the dominating

Electronic supplementary material The online version of this article (doi:10.1007/s00348-014-1808-3) contains supplementary material, which is available to authorized users.

W. Hubschmid $(\bowtie) \cdot$ A. Denisov

Paul Scherrer Institut, 5232 Villigen, Switzerland

e-mail: walter.hubschmid@psi.ch

F. Biagioli

Alstom Switzerland, 5242 Birr, Switzerland frequency of the sound, which is generated by the flame. This has been observed since some time. In particular, Giezendanner et al. (2003), Duan et al. (2005), Weigand et al. (2005), and Meier et al. (2007) reported on strong periodic movements of the inner and outer recirculation zones that are formed in the vicinity of the heat release zone of a swirl combustor. Hubschmid et al. (2002, 2008) and Tylli et al. (2005), on the other hand, reported on strong movements in a swirl combustor of the $\mathrm{OH}$ fields, as detected by LIF of OH (LIF: laser-induced fluorescence); however, these authors did not determine the flow field in order to clarify the origin of the movement of the $\mathrm{OH}$ fields, which can with some caution be regarded as flame movement.

In recent years, a large number of investigations were devoted to the study of the effect of sound on swirling flows. To a large extent, swirling reacting flows were thereby considered. Notably, Hauser et al. (2012) investigated the transformation of acoustic velocity into rotational flow oscillation in a swirl combustor. Wehe et al. (2011) determined experimentally the variation in the total heat release as response of the flame to acoustic perturbation in swirl combustors. Hosseini et al. (2012) made phaseresolved measurements of thermoacoustic effects in swirl burners, measuring flame and flow field by means of $\mathrm{OH}$ LIF and of PIV (PIV: particle image velocimetry), respectively. O'Connor and Lieuwen (2011) investigated the velocity and vorticity field in a swirl combustor, as disturbed by transverse external acoustic forcing. The same authors (O'Connor and Lieuwen 2012) reported on large variations of swirling flow by externally (transversely) irradiated sound; in particular, the effect of the sound in the planes normal to the main flow direction was investigated by decomposing the flow field in a sum of azimuthal modes. O'Connor and Lieuwen (2013), in the same 
arrangement, gave additional results of phase-resolved measurements (with respect to the acoustic forcing) for the vorticity and for the axial and the transverse velocity, along an axis in the subarea of the outer shear layer. General overviews on acoustic response in premixed swirl burners were given recently by Lawn et al. (2004) and by Candel et al. (2012). For general notions on thermoacoustic instabilities, one may consult Poinsot and Veynante (2005).

Schimek et al. (2012), in their investigation of the influence of external acoustic forcing at various intensities on flame and flow, reported results on concomitant variation in the flame angle. Actually, the periodic movement in the flow field, induced by the irradiated sound, may affect the thermoacoustic generation of sound in the case of combustion: If the amplitude of the movement of the gas is spatially dependent, the angle of the flame brush with respect to the burner axis may change, and therewith also the flame area. Thus, a change in the heat release rate may result.

In the present article, we investigate how the flow velocity field of non-reacting air downstream a swirl burner, measured by PIV, is modulated by sound, which is irradiated by loudspeakers that are positioned upstream the burner. The data of the PIV measurements of the flow field were evaluated in two ways. In the first approach, from the sequence of measured or primary images - consisting of about 2,700 single PIV measurements of an experimental run, which are represented by matrices of (two-dimensional) vectors of flow velocity - a set of secondary images was generated by the POD software (POD: proper orthogonal decomposition), which is implemented in the commercial PIV system used by us.

These secondary images are, in sequence, closest in average to the set of measured images under the condition of mutual orthogonality. They constitute a basis in the space of images, so that with appropriate coefficients, each of the primary images, which are obtained in the evolution of time, can be represented as a linear combination of these secondary images. In general, POD allows separating the quasistationary (or periodically varying) spatial configurations in a fluid from the randomly fluctuating configurations generated by turbulence. Furthermore, by a spectral decomposition of the time series of the coefficients for the various modes, the response of the fluid to an external perturbationi.e., in our case, the periodic forcing of the fluid by sound waves - can be visualized in a qualitative manner.

To obtain the main result of the present investigation, i.e., to relate quantitatively the total variation in the flow velocity in the combustor after the burner, caused by the acoustic irradiation, and the variation in the flow velocity from the variation in the acoustic velocity (see Sect. 3) alone, we defined subareas within the total cross section of the flow observed by PIV and determined the spatially averaged flow velocities in these subareas in dependence of the phase angle of the irradiated sound. Thus, local variations in the flow velocity generated by the sound irradiation were obtained by this second approach of evaluating the PIV.

Complementary to the experiments, large eddy simulations (LES) were performed, as a first step of validation of the assumptions employed in such simulations. For the general procedure of applying LES to combustion in swirling (reacting and non-reacting) flows, we refer to the earlier paper of Biagioli (2006). Some specific results from LES on stabilization mechanisms in swirling flows have recently been described by Biagioli et al. (2013).

\section{Experiment}

\subsection{Test rig}

The experiments described in this article were performed in a test rig for pre-premixed gas combustion. It contains a downscaled version of the Alstom EV burner (Döbbeling et al. 2005). For the investigations presented here, the test rig was operated with preheated air only. The air enters the burner from a tube of a diameter of $8 \mathrm{~cm}$. In two locations on opposite sides of this tube, at a distance of $63.5 \mathrm{~cm}$ before the exit of the burner, two loudspeakers are mounted. More downstream, in distances of $20 \mathrm{~cm}(\mathrm{P} 1), 30 \mathrm{~cm}$ $(\mathrm{P} 2)$, and $40 \mathrm{~cm}(\mathrm{P} 3)$ from the loudspeakers, there are ports where microphones can be inserted or which are blocked otherwise.

The tube downstream the burner has a diameter of $9 \mathrm{~cm}$. Windows out of quartz glass, just after the burner exit, allow optical measurements: two narrow windows (narrow with respect to the horizontal extension) for the laser light sheets and a window of $75 \mathrm{~mm} \times 75 \mathrm{~mm}$ for the observation of the PIV signal, using a CCD camera; see Fig. 1. In this tube, there are four other ports for microphones, in distances of $66 \mathrm{~cm} \mathrm{(P4),} 75 \mathrm{~cm}$ (P5), $84 \mathrm{~cm} \mathrm{(P6),} \mathrm{and}$ $93 \mathrm{~cm}$ (P7) from the loudspeakers. Figure 2 shows a scheme of the test rig. In this figure, only four ports for microphones are marked. This refers to the case of determination of the acoustic velocity upstream and as well downstream the burner; see the next subsection.

The exit of the EV burner has a diameter of about $4 \mathrm{~cm}$. This corresponds to a cross section of about $12.5 \mathrm{~cm}^{2}$. The flow velocities of the air, noted below, refer to this area. The actual cross section for the air flowing through the burner is given by the slits in the cone of the burner and is about $7.5 \mathrm{~cm}^{2}$ for the two slits together. Figure 1 shows the direction of the laser beam with respect to the EV burner and how the burner is positioned within the tube downstream the burner. A spatial view of the EV burner is given in the later Fig. 9. 


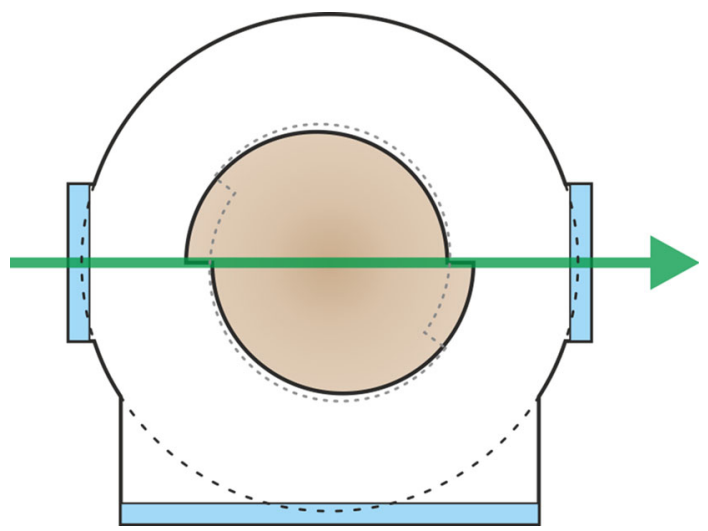

Fig. 1 Cross section through the tube downstream the burner. The double-cone EV burner is shown in brown color. The green arrow shows the direction of the light sheet. The quartz windows are indicated in blue. The large window, which is seen at the bottom, served for the observation of the scattered light by the PIV camera

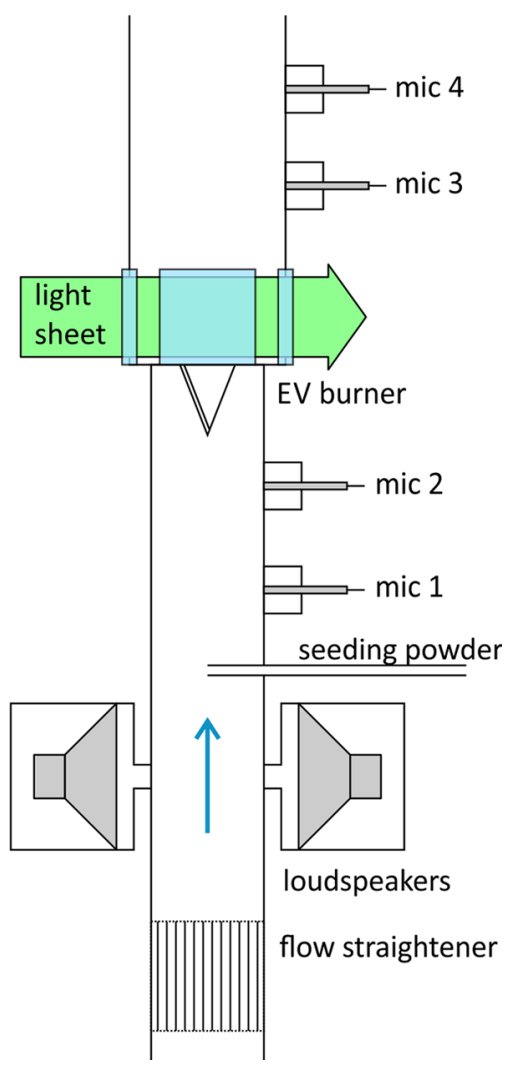

Fig. 2 Scheme of the test rig, showing the acoustic instrumentation for the determination of the acoustic velocity upstream and downstream the burner

\subsection{Acoustic instrumentation}

Microphones of the type Brüel\&Kjaer 4939 (cp. http:// www.bksv.com) were used to measure sound pressure and therewith to determine the acoustic velocity, see Sect. 3 . The amplified signal was sampled at $4 \mathrm{kHz}$ with a 16-bit
A/D converter. In the one experiment, two microphones each upstream and downstream the burner were positioned, to obtain upstream and downstream acoustic velocity. In other experiments, three microphones on one of the sides of the burner were used, in order to examine the accuracy of the acoustic measurements.

The power of the loudspeakers was $2 \times 50 \mathrm{~W}$. They generated sound pressures, at a flow velocity of $62 \mathrm{~ms}^{-1}$, of up to about $200 \mathrm{~Pa}$, depending on the sound frequency and on the position of the measurement. At the flow velocity of $31 \mathrm{~ms}^{-1}$, sound pressures up to about $400 \mathrm{~Pa}$ were generated. The higher sound pressures are attributed to the enlarged efficiency of the loudspeakers at lower flow velocities.

\subsection{Particle image velocimetry (PIV)}

To seed the air flow with particles for PIV measurements, a secondary air stream $(20 \mathrm{l} / \mathrm{min}$, about $2 \%$ of the main flow) was passed through a (fluidized bed) tube filled with $\mathrm{Al}_{2} \mathrm{O}_{3}$ powder (MARTOXID MR70, mean diameter about $0.8 \mu \mathrm{m}$ ). The particles were illuminated right above the burner exit by the beam of a high-repetition-rate Quantronix Darwin Duo Nd:YLF laser, which was stretched into a vertical sheet. The images were recorded with a LaVision HSS6 high-speed CCD camera, equipped with a Nikon $105 \mathrm{~mm}$ microlens.

The camera could acquire 2,728 PIV image pairs at a maximal rate of about $2,760 \mathrm{~Hz}$. Laser and camera were timed by a LaVision high-speed controller. The repetition rate of the laser and the acquisition rate of the camera were chosen to be 12 times the acoustic forcing frequency, which allowed us to resolve 12 phases of acoustic fluctuations (in steps of $30^{\circ}$ ) up to $230 \mathrm{~Hz}$. The start of the image acquisition was synchronized with the acoustic forcing; thus, the phase angles of each image with respect to the acoustic oscillation were defined. The analysis of the PIV data and the POD decomposition were done using the software LaVision Davis 7.2 using decreasing window size: first 64 by 64 and then 32 by 32 pixels with $50 \%$ overlap, resulting in 64 by 64 velocity fields with spatial resolution of about $1 \mathrm{~mm}$. These velocity fields were postprocessed using MATLAB.

\section{Acoustic forcing}

\subsection{General theory}

The acoustic pressure wave in the upstream part and as well in the downstream part of the test rig is assumed to be a non-dissipating plane wave. The general form of such plane wave, in domains of constant cross section, and when 
sound generation can be considered as negligible, is the sum of a forward and a backward propagating wave of the general form

$p(y, t)=A^{+}(t-y / c)+A^{-}(t+y / c)$,

where $A^{+}$and $A^{-}$are arbitrary functions; $y$ refers to the coordinate in direction of the wave propagation. In general, the temporal evolution of a plane non-dissipating acoustic wave is determined by measuring two independent functions of the time. This is usually done by measuring the time dependence of the acoustic pressure at two different positions.

From the equation of momentum

$\frac{1}{c^{2}} \frac{\partial p}{\partial t}+\rho_{0} \frac{\partial v}{\partial y}=0$

where $c$ is the speed of sound and $\rho_{0}$ is the time-averaged and spatially constant density of the medium, the following expression for the acoustic velocity $v$ can be derived as

$v(y, t)=\frac{1}{\rho_{0} c}\left(A^{+}-A^{-}\right)$.

The sound generated by the loudspeakers in our experiment is rather monochromatic. The sound generated by the flow, on the other hand, covers a broad frequency band; its intensity is, however, much smaller. In the (approximate) case of monochromatic sound (with angular frequency $\omega$ ), the temporal dependence of the sound pressure at some position $y_{i}$ is

$p_{i}=A_{i} \cos \left(\omega t+\alpha_{i}\right)$,

For two positions $y_{1}$ and $y_{2}$, we write this as

$p_{1}=A_{1} \cos \omega t, \quad p_{2}=A_{2} \cos (\omega t+\alpha)$,

and one obtains then the following expression for the acoustic velocity at an arbitrary position $y$ this assertion. In such case, according to Eq. (6), the determination of the acoustic velocity requires the measurement of the (harmonic) acoustic pressure at two different positions.

To this goal, we inserted into Eq. (6) the values of A1, $\mathrm{A} 2$, and $\alpha$ as obtained from the measurements of $p_{1}(t)$ and $p_{2}(t)$. The values of the amplitudes of the pressure oscillation at a given frequency were obtained from the intensity of the corresponding peak in the FFT spectrum of the recorded pressure. To determine the conversion factor, acoustic pressures were recorded for an intensity calibrator at $1,000 \mathrm{~Hz}$ and $10 \mathrm{~Pa}$.

In order to have some control on the accuracy of such determination of the acoustic velocity, we measured acoustic pressures $p_{1}(t), p_{2}(t)$ for different pairs $\left(y_{1}, y_{2}\right)$ of axial positions of the microphones, thereby we obtained identical results for the acoustic velocity $v(y, t)$ at some position $y$, within differences in the order of $10 \ldots 20 \%$. This shows that the (time-dependent) acoustic velocity as defined in Sect. 3.1 represents a well-defined physical quantity, clearly to be distinguished from the (total) variation in the flow velocity, as originating from the irradiation of sound.

The acoustic pressure spectra as obtained from measurements by microphones show peaks at the forcing frequency. The background signal around the forcing frequency was by about one and a half to two orders of magnitude smaller than the signal at the forcing frequency. This was checked for acoustic forcing at a large number of different forcing frequencies. In general, the amplitude at the peak of the forcing frequency was larger by the mentioned factor in the whole pressure spectrum, except in the region where sound intrinsically generated by the precession of the vortex core (PVC; see Syred 2006; Oberleithner et al. 2011) was registered.

$v(y, t)=\frac{\left\{A_{1} \cos \left[\omega\left(y-y_{2}\right) / c\right]-A_{2} \cos \alpha \cos \left[\omega\left(y-y_{1}\right) / c\right]\right\} \sin \omega t-A_{2} \sin \alpha \cos \left[\omega\left(y-y_{1}\right) / c\right] \cos \omega t}{\rho_{0} c \sin \left[\omega\left(y_{2}-y_{1}\right) / c\right]}$.

\subsection{Determination of acoustic velocity; intrinsically generated sound}

The acoustic measurements at our test rig were taken at temperatures of about $380{ }^{\circ} \mathrm{C}$. The flow velocity was $31 \mathrm{~ms}^{-1}$ for most experiments; some additional experiments were carried out at a flow velocity of $62 \mathrm{~ms}^{-1}$. We assumed thereby non-dissipating, approximately harmonic plane acoustic waves; see Sect. 4 for some validations of
This intrinsically generated sound had, for a flow velocity of $31 \mathrm{~m} / \mathrm{s}$, a frequency of about $650 \mathrm{~Hz}$. Its peak in the pressure spectrum-for a measurement with a microphone at position P4 (see Sect. 2.1)—was nearly as high as the one at the forcing frequency; also, the width of this peak, about $\pm 20 \mathrm{~Hz}$, was clearly larger than the one from forcing, determined by the spectral resolution of $1 \mathrm{~Hz}$. With microphones positioned at P5, P6, and P7, however, no additional peak from PVC was seen. This 
indicates that the sound generated by the PVC was mainly irradiated into a small horizontal segment, or in other words, the intensity of the sound from the PVC, propagating in axial direction, was so small that it was hidden by the noise generated by turbulence.

\section{Acoustic measurements in quiescent air: measurements by microphones and by PIV}

In order to test the accuracy of acoustic measurements by microphones close to the burner exit, where plane waves are only approximately given, we determined, in quiescent air, the acoustic velocity not only by measurements with microphones, but also with PIV. The microphones were positioned at distances of $11.5 \mathrm{~cm}$ and $29.5 \mathrm{~cm}$ from the burner exit. For the PIV measurements, aerosol particles formed from sulfur trioxide - using a tool manufactured by Dräger-were inserted into the volume above the burner exit. Figure 3 shows the result of a measurement by using this technique of seeding and with forcing at a frequency of $230 \mathrm{~Hz}$. One recognizes, in this image, how the plane wave evolves after the burner exit. For the distance of $6 \mathrm{~cm}$ from the burner exit, the amplitude of the acoustic velocity determined with the microphones was $v=0.85 \mathrm{~ms}^{-1}$, whereas PIV gave values of $v=1.0 \pm 0.1 \mathrm{~ms}^{-1}$. For the distance of $3.5 \mathrm{~cm}$ from the burner exit, the corresponding values were $v=0.85 \mathrm{~ms}^{-1}$ and $v=1.1 \pm 0.15 \mathrm{~ms}^{-1}$.

An evaluation of the statistical fluctuations for the PIV measurements for the data presented in Fig. 3-performed by comparing the sample of velocity vectors for $x=$ $-30 \ldots+30 \mathrm{~mm}$ and $y=55 \ldots 65 \mathrm{~mm}$-shows that the absolute values of $v$ vary by \pm 10 to $\pm 15 \%$ and that the directions of the vectors vary by about $\pm 10^{\circ}$. In consideration of the fact that the accuracy of the microphone measurements is of the order of $10 \ldots 20 \%$, see the previous section, we conclude that there is a good agreement between the determination of the acoustic velocity by microphones and by PIV. Furthermore, the PIV measurements show that the determination of the acoustic velocity by measurements with microphones in distances of about $10 \mathrm{~cm}$ and $30 \mathrm{~cm}$ from the burner exit, and using the assumption of plane non-dissipating waves, results in correct values, up to an error of $20 \ldots 30 \%$, for distances from the burner exit as small as about $3.5 \mathrm{~cm}$.

\section{Flow field under acoustic forcing measured by PIV}

\subsection{General flow pattern downstream the burner}

The flow downstream the burner has inner and outer recirculation zones; Fig. 4 shows the general flow pattern, as

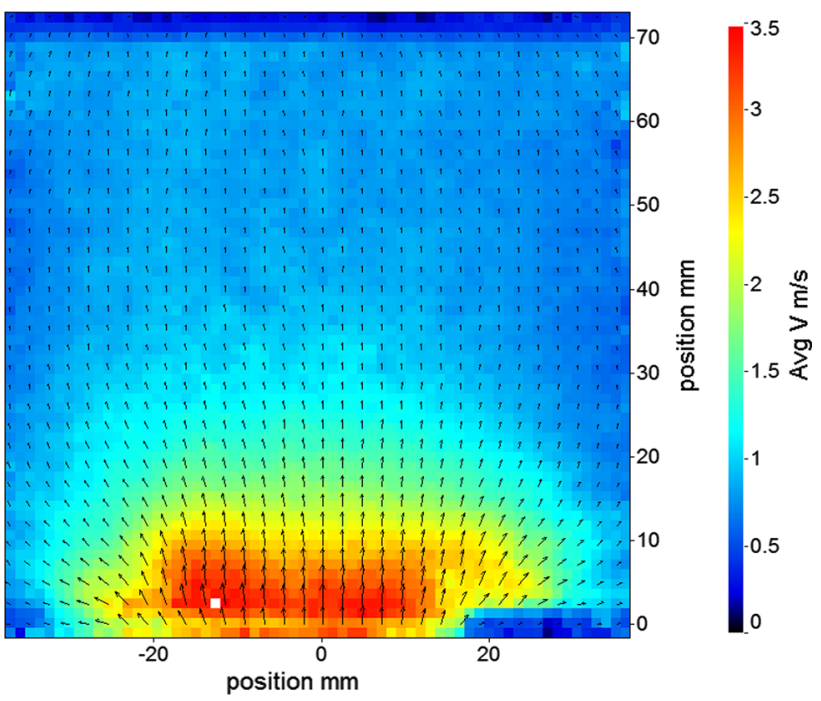

Fig. 3 Result of a PIV measurement of the (acoustic) flow field downstream the burner in quiescent air. One observes that already a few cms after the burner exit, an approximately plane wave is evolving. The arrows represent the amplitude of the oscillating flow velocity. The image shown was taken at the phase with maximum velocity in positive $y$-direction

observed with PIV measurements. This picture was obtained for a flow velocity of $31 \mathrm{~ms}^{-1}$ and a sound forcing of $230 \mathrm{~Hz}$ by averaging over all the images obtained in one run, i.e., averaging over the $12 \times 227$ images for the 12 acoustic phases considered. One observes some left-right asymmetry: The axis of the (broken) symmetry is about $2 \mathrm{~mm}$ to the left of the burner axis. Also, position and shape of the recirculation zones are not left-right symmetric. We attribute these deviations to the asymmetric cross section due to the window for the camera (cp. Fig. 1).

A control measurement for a flow velocity of $62 \mathrm{~ms}^{-1}$ showed about the same general pattern as the experiment with flow velocity of $31 \mathrm{~ms}^{-1}$; in particular, the position of the center point of the central recirculation zone (CRZ) varied only by a distance of about $1 \mathrm{~mm}$. In the absence of sound irradiation, at otherwise unchanged operation conditions, changes in the positions of the recirculation zones of a few mm were observed. This is, however, not to be considered as significant, as positions of center points of recirculation zones may change by distances of the order of about $3 \mathrm{~mm}$ just by variation in some of the evaluation parameters for the analysis of the PIV data, as given in the commercial software.

In order to empirically study dynamical properties of the flow, a proper orthogonal decomposition (POD; see Sect. 1), according to the general scheme

$\vec{v}\left(\vec{x}, t_{j}\right)=\overrightarrow{\vec{v}}(\vec{x})+\sum_{i} c_{i}\left(t_{j}\right) \vec{\phi}_{i}(\vec{x})$

was applied to the set of the matrices of vectors of flow velocity obtained by PIV in one experimental run. 
The spatial functions $\vec{\phi}_{i}(\vec{x})$ represent the basis of the POD. Spatial configurations in the gas flow, which are partly conserved (or periodically varying with some frequency), can be recognized in the first few modes. Figure 5 shows the spatial dependence of the first three modes, as obtained from an experiment with acoustic forcing at $230 \mathrm{~Hz}$ and flow velocity of $31 \mathrm{~ms}^{-1}$. The spatial function $\vec{\phi}_{1}(\vec{x})$ of mode 1 has a pronounced antisymmetry with respect to the $y$-axis, characteristic for a mode that is dominated by PVC, whereas mode 3 is rather symmetric with respect to the $y$-axis, characteristic for a mode that is dominated by the sound forcing.

For forcing at other frequencies, the functions $\vec{\phi}_{i}(\vec{x})$ show in part some accord. In general, however, they

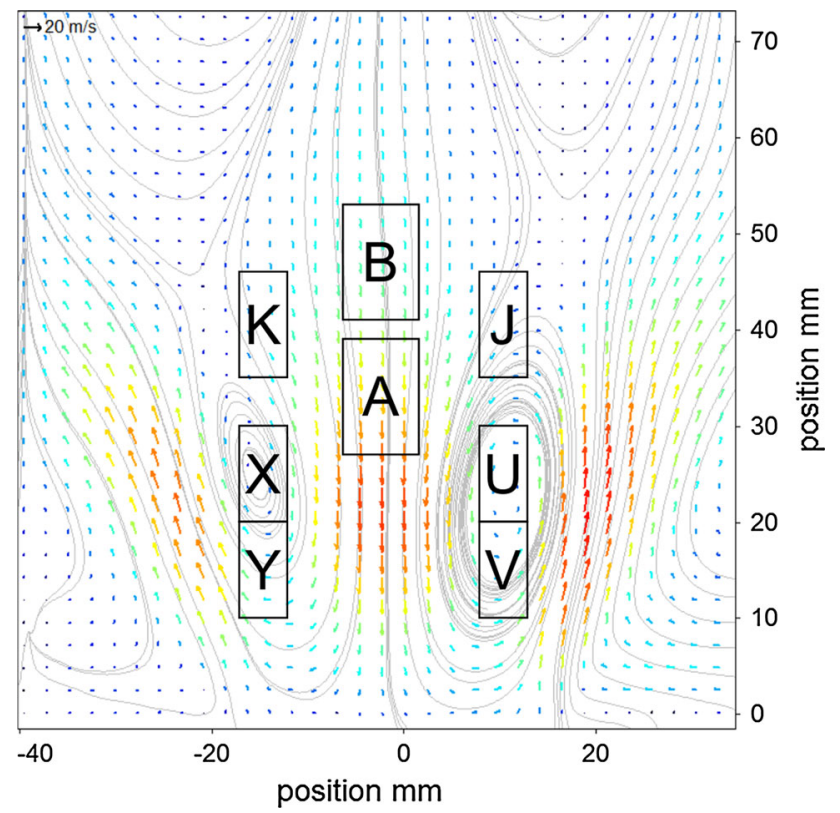

Fig. 4 PIV image of overall average flow field (including averaging over acoustic phases) downstream the burner for a flow velocity of $31 \mathrm{~m} / \mathrm{s}$ and a sound forcing with a frequency of $230 \mathrm{~Hz}$. Inner and outer recirculation zones in the flow field are recognized. The various subareas for the local analysis of phase-averaged PIV (cp. Fig. 8) are depicted disagree rather strongly; also, the spatial mode functions, which are obtained from the results of experiments with acoustic forcing and without any forcing, agree only partially. This is not surprising, as each POD refers to the specific sample of experimental data that are obtained in some experimental run.

The data of each experimental picture, labeled in their temporal sequence by the index $t_{j}$, are represented as a linear combination of the basis spatial functions $\vec{\phi}_{i}(\vec{x})$ with coefficients $c_{i}\left(t_{j}\right)$, as given explicitly in Eq. (7) and depending on the discrete time variable $t_{j}$. The spectral decomposition, up to mode 12 , of the functions $c_{i}\left(t_{j}\right)$, for the same measurement where spatial functions $\vec{\phi}_{i}(\vec{x})$ are shown in Fig. 5, is depicted in Fig. 6. One observes that many of the modes have a (sharp) spectral peak at the forcing frequency of $230 \mathrm{~Hz}$. Other modes have a peak around the PVC frequency at about $650 \mathrm{~Hz}$. Further experiments, operating the test rig for flow velocities between 8 and $62 \mathrm{~ms}^{-1}$, showed about a linear dependence of the PVC frequency on the flow velocity.

In addition, some of the modes have contributions at the difference in these two frequencies. Barely visible are also contributions at the sum frequency of these frequencies. They both refer to nonlinear interactions between the two oscillations. A further experiment under same conditions but with a forcing frequency of $180 \mathrm{~Hz}$ shows a peak at this frequency and also a peak at the frequency of $650 \mathrm{~Hz}$.

In comparison, the spectral content of the first modes without any acoustic forcing is shown in Fig. 7. It shows that most modes again have a peak at the intrinsic frequency at about $650 \mathrm{~Hz}$. Other peaks in the spectra are not observed. Some kind of enhanced noise only at low frequencies is seen for modes 3 and 4 .

\subsection{Periodic variation in flow by acoustic forcing}

The variation in the flow velocity field by the acoustic forcing is obtained from the comparison of the averaged
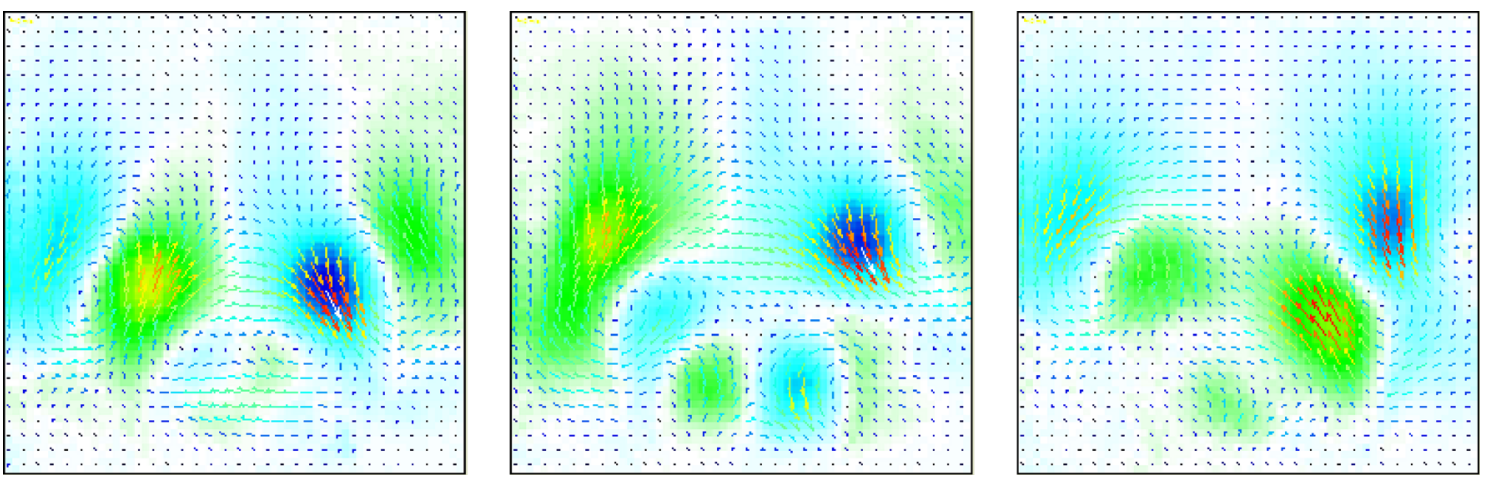

Fig. 5 Spatial functions $\vec{\phi}_{i}(\vec{x})$ of the first three POD modes, which are obtained from the data of a PIV measurement at the flow velocity of $31 \mathrm{~m} / \mathrm{s}$ and a forcing frequency of $230 \mathrm{~Hz}$. The colors represent the axial component of the flow velocity $\left(\right.$ green: $v_{y}>0$, blue: $\left.v_{y}<0\right)$ 
Fig. 6 Spectral decomposition of the coefficient functions $c_{i}\left(t_{j}\right)$ (from below) for the first 12 modes, which are extracted from the data of a PIV measurement at the flow velocity of $31 \mathrm{~m} / \mathrm{s}$ and a forcing frequency of $230 \mathrm{~Hz}$. Besides the peak at the frequency of the sound forcing, in many of the modes, a further peak at the frequency of around $650 \mathrm{~Hz}$ is observed. For some modes, peaks at the difference frequency and rudiments of a peak at the sum frequency are also seen

Fig. 7 Spectral decomposition of the coefficient functions $c_{i}\left(t_{j}\right)$ for the first 12 modes, which are extracted from the data of a PIV measurement at the flow velocity of $31 \mathrm{~m} / \mathrm{s}$ and without acoustic forcing. In many of the modes, one observes a peak at the frequency of around $650 \mathrm{~Hz}$
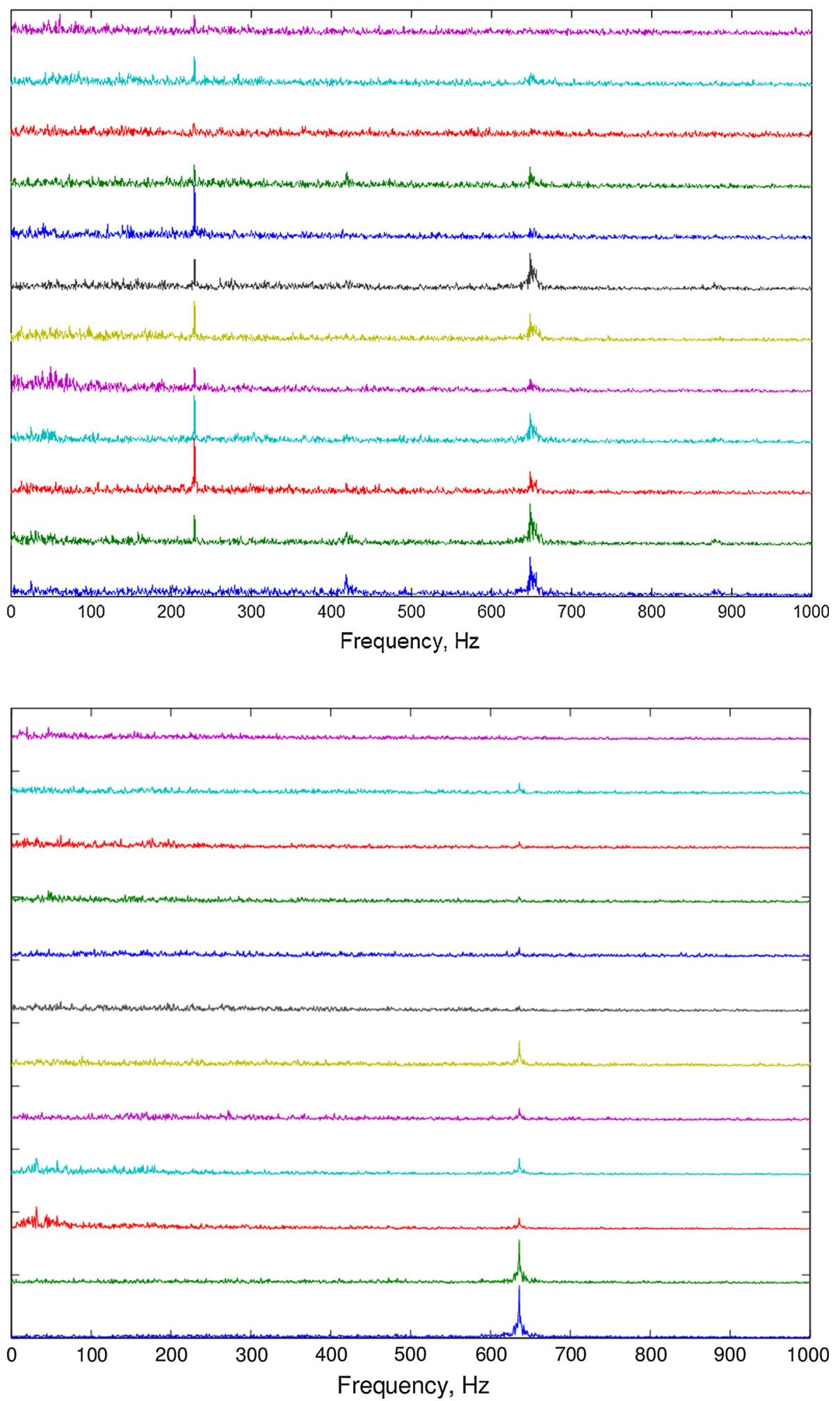

single PIV measurements for any of the 12 considered phase angles of the sound forcing. To depict this in a most illustrative way, the flow velocity vectors (for any of the 12 acoustic phase angles) were averaged over some selected subareas of the whole field observed. In this way, the statistical fluctuations from turbulence could be further reduced.

The location of the selected subareas is shown in Fig. 4. Subareas A, B, J, and $\mathrm{K}$ are within the validity of the planewave assumption, i.e., the deviations of amplitudes of the 

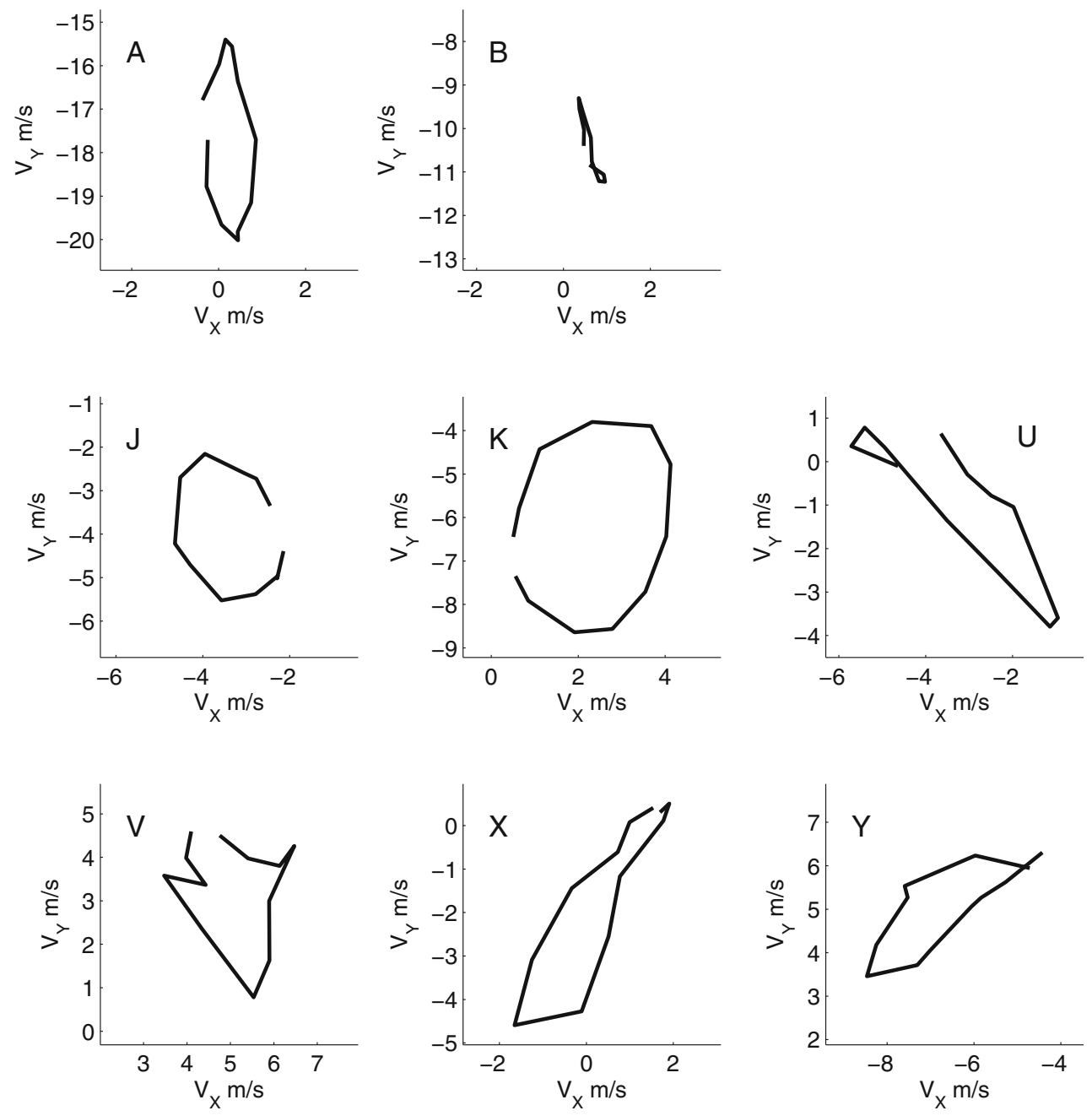

Fig. 8 Phase-dependent local variation in flow field (for 12 phases) for eight subareas of the PIV measurements as defined in Fig. 4. The vertices in the diagrams refer to steps of $30^{\circ}$ in phase angles of sound, at which the measurements were taken. Flow velocity was $31 \mathrm{~m} / \mathrm{s}$,

flow velocity, with respect to the plane-wave assumption, are in the order of not more than $20 . .30 \%$. For the other subareas, they are somewhat larger, see Fig. 3. This, however, will modify only modestly the general result given in the next paragraph but one.

Figure 8 shows the result for the various selected subareas for an experiment with flow velocity of $31 \mathrm{~ms}^{-1}$ and with forcing of sound with a frequency of $230 \mathrm{~Hz}$. Standard deviation of the mean values (with respect to spatial averaging on the subarea) for $v_{x}$ (for the various phases) was in the order of $0.5 \ldots 0.6 \mathrm{~ms}^{-1}$, and for $v_{y}$ in the order of $0.5 \ldots 0.7 \mathrm{~ms}^{-1}$, depending on the observed subarea. (Statistical deviations of the mean value for the temporal averaging on the 227 measurements per phase are higher, of the order of $1 \mathrm{~m} / \mathrm{s}$.) These fluctuations originate from turbulence, and they are supposed to cause the deviations from a supposed more regular behavior of the variation in $\vec{v}$ with the acoustic phase. and the forcing frequency was $230 \mathrm{~Hz}$. The scales on the coordinate axes refer to the components $v_{x}$ and $v_{y}$ of the average flow velocity in the subareas. The sense of rotation is clockwise for $\mathbf{A}, \mathbf{K}, \mathbf{U}, \mathbf{V}, \mathbf{X}$, and it is counterclockwise for $\mathbf{J}$ and $\mathbf{Y}$

Looking in detail at the results for the subareas, one observes that the flow reacts on the acoustic forcing at full power of the loudspeakers with variations in the flow velocity of the order of $\pm 2 \mathrm{~ms}^{-1}$ in the axial $y$-direction (with exception of $\mathrm{B}$ ); variations in the flow velocity in the $x$-direction differ strongly and are mostly smaller. These variations in the amplitude of the flow velocity during an acoustic period may be compared with values of the acoustic velocity obtained from measurements by microphones as described in Sect. 3. For the experiment in discussion, we obtained values of about $\pm 0.6 \mathrm{~ms}^{-1}$ (at the position $y=0.06 \mathrm{~m}$ above the burner exit). For the flow velocity of $62 \mathrm{~ms}^{-1}$, the corresponding data were approximately \pm 1 and $\pm 0.2 \mathrm{~ms}^{-1}$ for flow movement and acoustic velocity, respectively. Further experiments show that there is an approximately linear dependence of the variations in the flow velocity with variations in the acoustic pressure at otherwise unchanged conditions. 

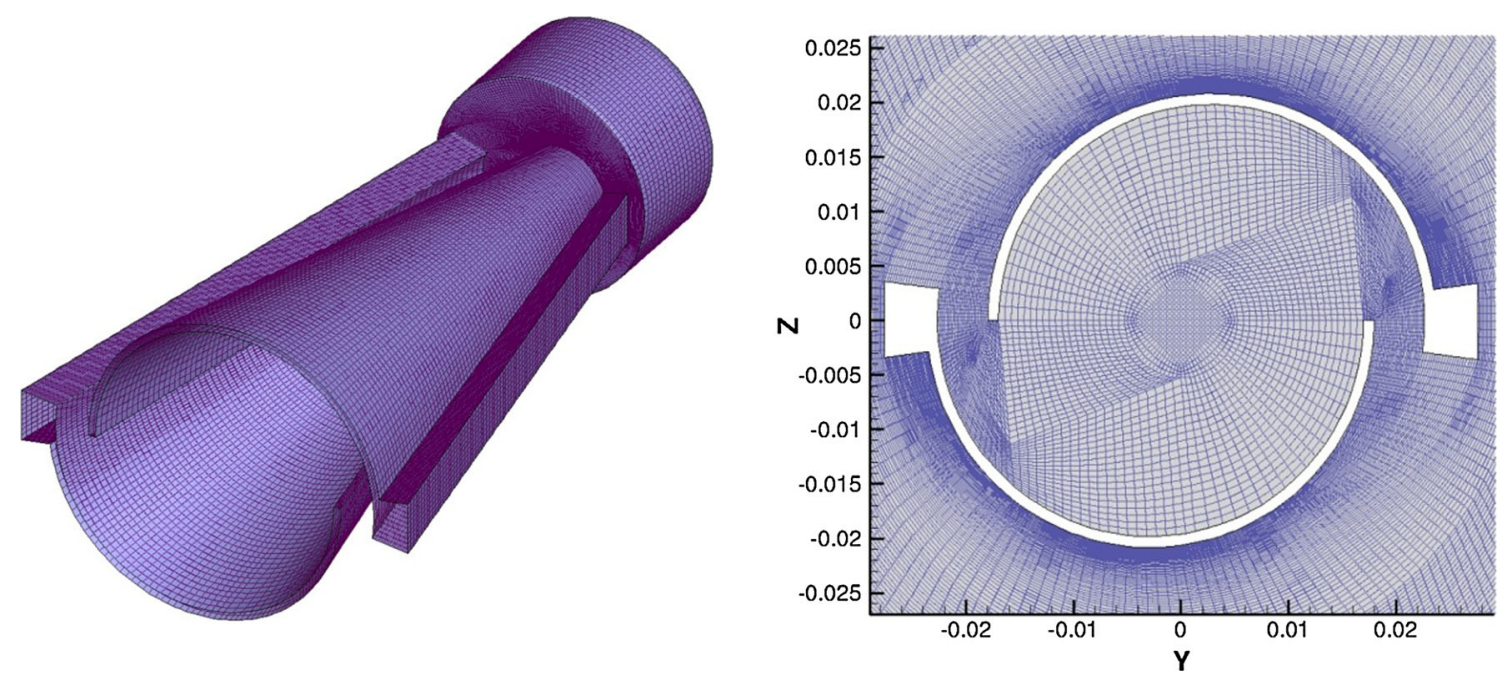

Fig. 9 Spatial view and cross section of the EV burner; the grid used in the numerical simulation is shown

Considering the sense of rotation of the variation in the flow velocity with respect to the acoustic phase, the following statements can be done: As is expected by rotational symmetry with respect to the burner axis, the sense of rotation is changing from clockwise to counterclockwise between subareas on the left and on the right of the burner axis. (Subarea U vs. X does not obey this rule. Both have a clockwise dependence on the acoustic phase.) The sense of rotation is also changed by going more upstream, i.e., from subarea $\mathrm{J}$ to $\mathrm{V}$ and from subarea $\mathrm{K}$ to $\mathrm{Y}$, respectively. An additional evaluation for a subarea between $\mathrm{K}$ and $\mathrm{X}$ shows also a clockwise sense of rotation, whereas an intermediate subarea between $\mathrm{J}$ and $\mathrm{U}$ does not give a well-defined result with respect to the sense of rotation. A sense of rotation seems is also recognizable for subarea A, although it covers an area, which is lying rather symmetrically with respect to the vertical symmetry axis.

\section{Numerical simulation by LES and comparison with experiment}

Large eddy simulation of the burner-combustor setup was done by means of the commercial CFD Solver Fluent. This code is based on treating the flow as incompressible and by using a standard Smagorinsky closure of turbulent transport with a Smagorinsky constant $C_{\mathrm{s}}=0.1$; see Smagorinsky (1963). The numerical discretization of the momentum equation consists in generating second-order central differences for the gradient diffusion terms and bounded central differences for the convection terms; see Jasaka et al. (1999). Hereto, a block structured hexa-grid of approximately $3 \times 10^{6}$ elements was generated. A scheme of the grid for burner and its surrounding is shown in Fig. 9. To simplify the simulation, the combustor was treated as having rotational symmetry, neglecting thereby the deviation from such symmetry caused by the optical windows (cp. Fig. 1). The LES snapshots (in intervals of $10^{-5} \mathrm{~s}$ ) of the transient solution for the velocity components are registered for two mutually orthogonal planes with the symmetry axis as common axis.

The flow response to the acoustic perturbation is obtained from a superposition of a broadband noise variation $v^{\prime}\left(t_{k}\right) \equiv v_{k}^{\prime}$ at the inlet of the computational domain to the undisturbed flow. This allows the calculation of the effect of irradiated sound on the flow for a whole spectrum of frequencies in one computational run. On the other hand, this renders the evaluation for forcing of sound at a specific frequency somewhat more difficult, as such information has to be extracted from the broadband excitation. To proceed here, LES snapshots sampled every four time steps were analyzed by means of a POD: The snapshots are represented, as in the case of the experimental data, by a time-dependent linear combination of POD modes $\vec{\phi}_{i}(\vec{x})$ indexed by $i$. The corresponding time-dependent coefficients $c_{i}\left(t_{j}\right)$ are obtained from projecting each transient spatial snapshot on the sequence of POD modes.

We consider now a specific mode $i$. The related time sequence $c\left(t_{j}\right)$ contains two contributions: a first pure turbulent one (noise) $\varepsilon_{j}^{\prime}$ and a second dynamic one driven by the imposed broadband forcing of mass flow rate. According to standard control theory, the second contribution can be expressed as the convolution of the unit impulse responses $h_{1}(l=1,2, \ldots L$; at the sampling frequency of four LES time steps, i.e., in time steps of $4 \times 10^{-5} \mathrm{~s}$ ) with the perturbation $v^{\prime}$. The coefficients $c\left(t_{j}\right)$ can therefore be expressed as 
$c\left(t_{j}\right)=\sum_{l=1}^{L} h_{l} v_{j-l}^{\prime}+\varepsilon_{j}^{\prime} ; \quad j=1,2, \ldots, N$.

The discrete unit impulse response $h_{1}$ is obtained using the Wiener-Hopf method (substantially a least mean square error estimation method) based on the minimization of the function

$E\left(h_{1}, h_{2}, \ldots\right) \equiv \sum_{j=1}^{N}\left(c\left(t_{j}\right)-\sum_{l=1}^{L} h_{l} v_{j-l}^{\prime}\right)^{2}$

This is done, according to the rule

$\min E \rightarrow \frac{\partial E}{\partial h_{k}}=0, \quad k=1,2, \ldots, L$.

In the simulations performed, the inlet of the computational domain was placed $160 \mathrm{~mm}$ upstream the burner exit, $L$ was set equal to 40 , and the number $N$ of snapshots for the LES calculation was set equal to 3,000.

The Fourier transform $H_{i}(\omega)$ of the impulse responses $h_{l}$ is the transfer function (of the considered mode $i$ ) for the induced acoustical disturbance on the flow. Its value for a given $\omega$ is the response of the flow in linear regime to a harmonic perturbation of the inlet signal at frequency $\omega$. By linearly combining the POD modes according to coefficients given by the transfer functions $h_{i}(\omega)$, the response of the flow field to harmonic forcing $u^{\prime} \cos \omega t$ is obtained as

$$
\begin{gathered}
\vec{v}(\bar{x}, t)=\overrightarrow{\vec{v}}(\vec{x})+v^{\prime} \sum_{i}\left|H_{i}(\omega)\right| \cos \left(\omega t+\delta_{i}(\omega)\right) \vec{\phi}_{i}(\vec{x}), \\
H_{i}(\omega) \equiv\left|H_{i}(\omega)\right| \exp \left(i \delta_{i}\right)
\end{gathered}
$$

Note that in Eq. (11), the contribution of the turbulent part to the flow field is not included. It is shown in the literature (Davis 1979) that the procedure just described is well in line with the one based on harmonic excitation of single frequencies.

The comparison between LES and experiments with harmonic excitation at specific frequencies is done on the basis of Eq. (11). Some of the results of a simulation for a flow velocity of $31 \mathrm{~m} / \mathrm{s}$ and an excitation with sound of a frequency of $230 \mathrm{~Hz}$ are shown in Figs. 10 and 11. The time-averaged flow is depicted in Fig. 10. The recirculation zones in the time-averaged flow field of simulation and experiment differ somewhat in position and as well in extension. In addition, the velocity of the backflow in the center is clearly smaller in simulation than in experiment. Therefore, for local flow movements, caused by the acoustic forcing, only a semiquantitative comparison between experiment and simulation is possible. The limitations of the validity of the simulation arise possibly because the results of the calculations depend rather strongly on input parameters. Future investigations should

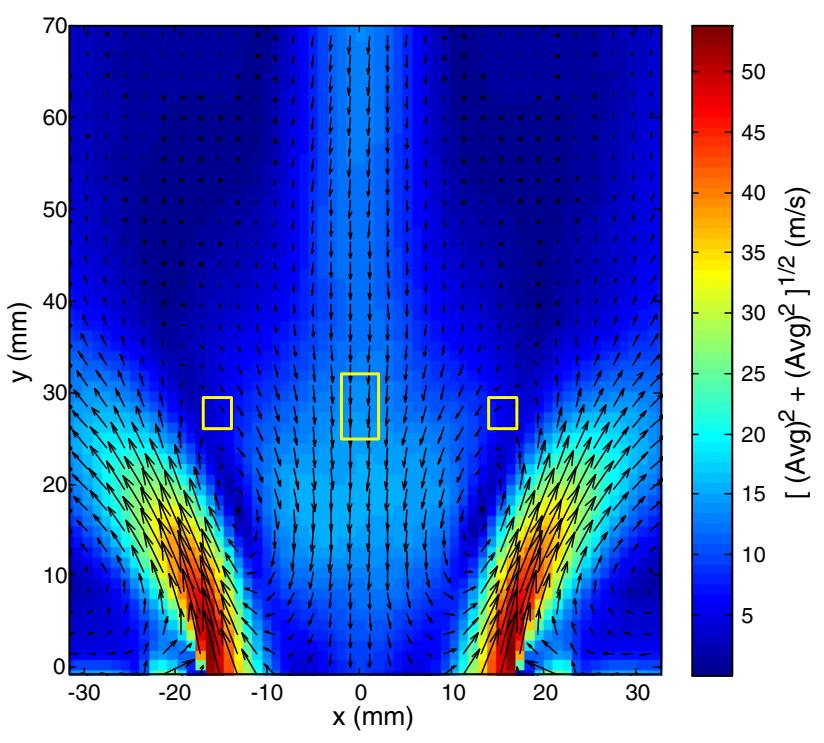

Fig. 10 Average flow field downstream the burner as obtained by LES. The rectangles refer to the areas for which the local and phasedependent variations in the flow are depicted in Fig. 11

clarify on this point. Simulation reveals also an intrinsically generated frequency, which is interpreted as caused by the precession of the vortex core; it differs in frequency by about $15 \%$ compared with the experimental value.

For the three areas marked by rectangles in Fig. 10, the spatially averaged variation in the flow (as a function of the acoustic phase) was determined; see Fig. 11. These areas ("left," "middle," and "right") were chosen as equivalents, with respect to the positions of the recirculation zones, to the areas $\mathrm{K}, \mathrm{B}$ and $\mathrm{A}$, J, respectively, which had been chosen for the data analysis of the PIV measurements. Other choices of areas for the LES evaluation gave similar results as the ones presented in Fig. 11.

In qualitative terms, there is reasonable accord between the results obtained from the PIV measurements and the simulations. The variations in the flow velocity with the phase of sound obtained in the simulations are of the same order as in experiment. Numerical simulation reproduces also the phase shift of nearly $\pi$ between the axial motion in the center and in the zones of the shear layer of the flow field after the burner, as was observed by PIV. This general result is recognized in accompanying GIF files of both experimental and numerical evaluations of sound forcing of swirling flow, as considered in the present investigation. The files are part of the online version of the paper and present the flow field downstream the burner for forcing of sound with frequency of $180 \mathrm{~Hz}$ and $230 \mathrm{~Hz}$, respectively, and for a flow velocity of $31 \mathrm{~m} / \mathrm{s}$. Due to deficient data of PIV in some regions, the area shown in the PIV files is somewhat reduced compared to the LES files. 

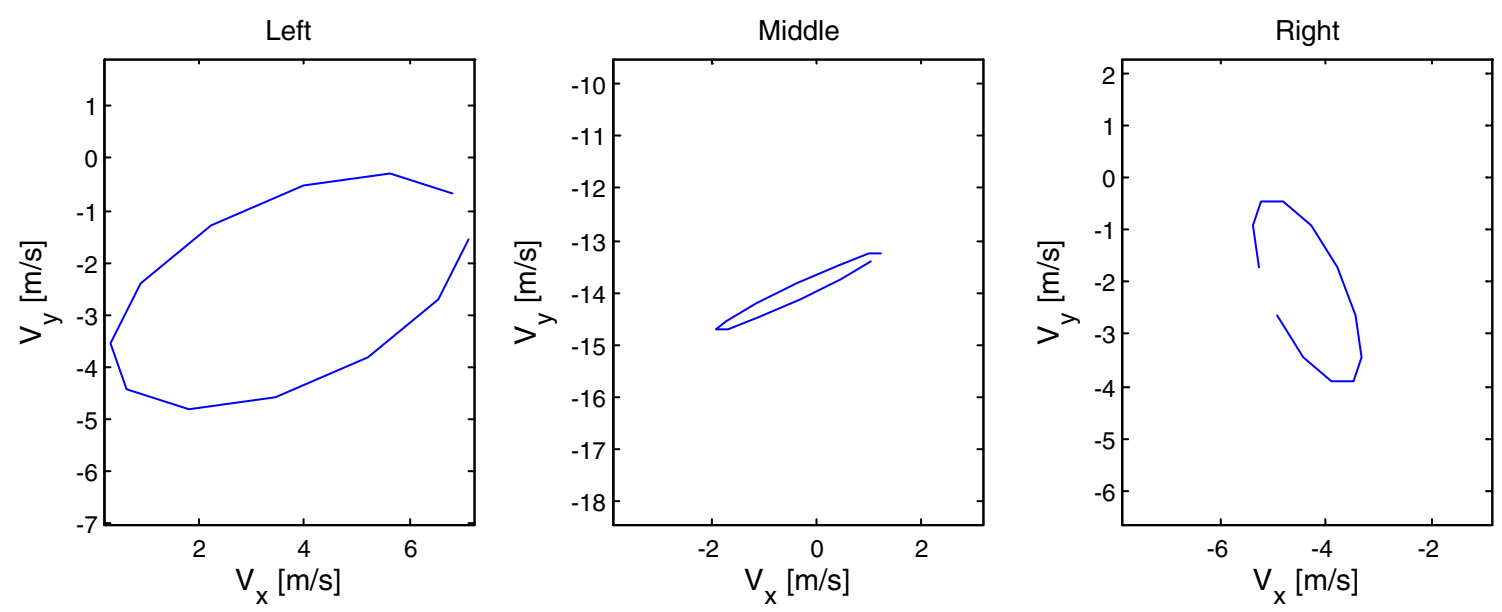

Fig. 11 Variation in the flow velocity with the acoustic phase in the areas of the rectangles as noted in the previous Fig. 10. Sense of rotation of the variation of the flow velocity, from left to right, is clockwise, (counterclockwise), counterclockwise

\section{Summary and outlook}

We investigated experimentally and numerically the effect of externally generated sound on non-reactive flow in a swirl combustor. The acoustic perturbation causes a variation in the flow field that is much larger than the variation in the velocity by the sound itself; the effect of the sound on the flow is thus amplified for a swirl flow such as given in our experiments. We observed also a nonlinear interaction between the intrinsically generated sound from the precession of the vortex core and the externally irradiated sound. Numerical simulation, using LES under the condition of incompressibility, was in addition performed. The effect of the sound irradiation was thereby modeled by periodic variation in the air flow at the inlet of the computational domain. Such calculation reproduces in part the effect of sound on the flow as observed experimentally. The present paper gives results of a first step within a systematic investigation of thermoacoustic effects in a swirl burner, with the general goal of improving and validating their numerical simulation.

Acknowledgments We thank Vishal Toro for building up the test rig at PSI and Rafal Slefarski for doing first tests. For maintaining and continuous improving of the test rig, we thank Pascal Beerkircher and Philipp Schmitt. For assistance in performing the LES simulations, we thank Federico Olimpi. Furthermore, we thank P. Jansohn for careful reading of the text and $\mathrm{Y}$. Mantzaras for discussions.

\section{References}

Biagioli F (2006) Stabilization mechanism of turbulent premixed flames in strongly swirled flows. Combust Theory Model 10:389-412

Biagioli F, Paikert B, Genin F, Noiray N, Bernero S, Syed K (2013) Dynamic response of turbulent low emission flames at different vortex breakdown conditions. Flow Turbul Combust 90:343-372
Candel S, Durox D, Schuller T, Palies P, Bourgouin JF, Moeck JP (2012) Progress and challenges in swirling flame dynamics. Compte Rendus Méc 340:758-768

Davis JH (1979) Wiener-Hopf methods for open-loop unstable distributed systems. SIAM J Control Optim 17:713-728

Döbbeling K, Hellat J, Koch H (2005) 25 years of BBC/ABB/Alstom lean premix combustion technologies. In: Proceedings of the ASME Turbo Expo (Paper GT2005-68269)

Duan XR, Meier W, Weigand P, Lehmann B (2005) Phase-resolved laser Raman scattering and laser Doppler velocimetry applied to periodic instabilities in a gas turbine model combustor. Appl Phys B80:389-396

Giezendanner R, Keck O, Weigand P, Meier W, Meier U, Stricker W, Aigner M (2003) Periodic combustion instabilities in a swirl burner studied by phase-locked planar laser-induced fluorescence. Combust Sci Technol 175:721-741

Hauser M, Wagner M, Sattelmayer T (2012) Transformation of transverse acoustic velocity of the burner approach flow into flame dynamics. In: Proceedings of ASME Turbo Expo 2012: GT2012-69051

Hosseini SMR, Gardner C, Lawn C (2012) The non-linear thermoacoustic response of a small swirl burner. Combust Flame 159:1909-1920

Hubschmid W, Inauen A, Bombach R, Kreutner W, Schenker S, Zajadatz M, Motz C, Haffner K, Paschereit CO (2002) Sound generating flames of a gas turbine burner observed by laserinduced fluorescence. PSI Scientific Report 2001/Volume V: 57-58. http://cdg.web.psi.ch/

Hubschmid W, Bombach R, Inauen A, Güthe F, Schenker S, Tylli N, Kreutner W (2008) Thermoacoustically driven flame motion and heat release variation in a swirl-stabilized gas turbine burner investigated by LIF and chemiluminescence. Exp Fluids 45:167-182

Jasaka HH, Weller HG, Gosman AD (1999) High resolution NVD differencing scheme for arbitrarily unstructured meshes. Int J Number Methods Fluids 31:431-449

Lawn CJ, Evesque S, Polifke W (2004) A model of the thermoacoustic response of a premixed swirl burner, part I: acoustic aspects. Combust Sci Technlo 178:1331-1368

Meier W, Weigand P, Duan XR, Giezendanner R (2007) Detailed characterization of the dynamics of thermoacoustic pulsations in a lean premixed flame. Combust Flame 150:2-26 
O'Connor J, Lieuwen $T$ (2011) Further characterization of the disturbance field in a transversely excites swirl-stabilized flame. In: Proceedings of ASME Turbo Expo 2011: GT2011-45221

O'Connor J, Lieuwen T (2012) Influence of transverse acoustic modal structure on the forced response of a swirling nozzle flow. In: Proceedings of ASME Turbo Expo 2012: GT2012-70053

O'Connor J, Lieuwen T (2013) Disturbance field characteristics of a transversely excited burner. Combust Sci Technol 183:427-443

Oberleithner K, Sieber M, Nayeri CN, Paschereit CO, Petz C, Hege HC, Noack BR, Wygnanski I (2011) Three-dimensional coherent vortex breakdown: stability analysis and empirical mode construction. J Fluid Mech 679:383-414

Poinsot T, Veynante D (2005) Theoretical and numerical combustion, 2nd edn. Edwards, Philadelphia

Schimek S, Cosic B, Moeck JP, Terhaar S, Paschereit CO (2012) Amplitude-dependent flow field and flame response to axial and tangential velocity fluctuations. In: Proceedings of ASME Turbo Expo 2012: GT2012-69785
Smagorinsky J (1963) General circulation experiments with the primitive equations. Mon Weather Rev 91:99-164

Syred N (2006) A review of oscillation mechanisms and the role of the precessing vortex core (PVC) in swirl combustion systems. Prog Energy Combust Sci 32:93-161

Tylli N, Hubschmid W, Inauen A, Bombach R, Schenker S, Güthe F, Haffner K (2005) Flame motion in gas turbine burner from averages of single-pulse flame fronts. PSI Scientific Report 2004/Volume V: 65-66. http://cdg.web.psi.ch/

Wehe SD, Li H, McManus KR (2011) Flame transfer function measurements in a single nozzle combustor. In: Proceedings of ASME Turbo Expo 2011: GT2011-45323

Weigand P, Meier W, Duan XR, Giezendanner R, Meier U (2005) Laser diagnostic study of the mechanism of a periodic combustion instability in a gas turbine model combustor. Flow Turbul Combust 75:275-292 